Research Paper

\title{
MUC7 Level As A New Saliva Risk Factor For Dental Caries In Adult Patients
}

\author{
Anna K. Szkaradkiewicz-Karpińska ${ }^{1}$, Anna Ronij ${ }^{1}$, Olga Goślińska-Kuźniarek², Izabela Przybyłek², Andrzej \\ Szkaradkiewicz ${ }^{2 凶}$ \\ 1. Department of Preclinical Conservative Dentistry and Preclinical Endodontics, University of Medical Sciences in Poznan, Poland \\ 2. Department of Medical Microbiology, University of Medical Sciences in Poznan, Poland \\ $\square$ Corresponding author: Prof. Andrzej Szkaradkiewicz, Department of Medical Microbiology, University of Medical Sciences in Poznan, Wieniawskiego 3, Str., \\ 61-712 Poznan, Poland. E-mail: szkaradkiewicza@poczta.onet.pl \\ (C) Ivyspring International Publisher. This is an open access article distributed under the terms of the Creative Commons Attribution (CC BY-NC) license \\ (https://creativecommons.org/licenses/by-nc/4.0/). See http://ivyspring.com/terms for full terms and conditions.
}

Received: 2018.08.06; Accepted: 2018.12.04; Published: 2019.01.01

\begin{abstract}
Purpose: Data currently available indicate the significance of salivary mucins MUC5B and MUC7 in the protection of teeth against caries. Our study aimed to determine the relationship between dental caries in adults and levels of MUC5B and MUC7.

Methods: The studies were conducted on 45 adult subjects selected on the basis of dental examination and calculation of the DMFT (Decayed, Missing, and Filled Teeth) index. Among these patients, two research groups were distinguished: group 1 included 19 caries-free subjects (DMFT $=0$ ); and group 2 included 26 patients with severe caries (DMFT > 13.9). Samples of whole unstimulated saliva were collected and centrifuged. MUC5B and MUC7 content in saliva supernatant were estimated using an enzyme-linked immunosorbent sandwich assay (ELISA). Analysis of the obtained data receiver operating characteristic (ROC) curves was employed to define relationships between the contents of the studied mucins and the detected dental caries.

Results: In subjects from group 1, the mean level of MUC5B amounted to $0.63 \pm 0.35 \mathrm{ng} / \mathrm{ml}$ and this was significantly higher than the concentration of mucin in patients of group 2 , which amounted to $0.38 \pm 0.32 \mathrm{ng} / \mathrm{ml}(p=0.023)$. The mean level of MUC7 amounted to $5.47 \pm 1.18 \mathrm{ng} / \mathrm{ml}$ and this was significantly higher than the level of the mucin in group 2 , which was $1.39 \pm 0.86 \mathrm{ng} / \mathrm{ml}(p<0.0001)$. In parallel, a relationship was detected between levels of the examined mucins and manifestation of dental caries. For MUC7, the optimal cut-off value was obtained (i.e. corresponding to $100 \%$ sensitivity and specificity), amounting to $2.5 \mathrm{ng} / \mathrm{ml}$ for the detection of dental caries risk.

Conclusions: Development of dental caries is linked to reduced concentrations of MUC5B and MUC7. The level of MUC7 may represent a significant parameter clinically suitable for evaluation of disease risk.
\end{abstract}

Key words: Oral health, saliva mucins, MUC5B, MUC7, dental caries.

\section{Introduction}

The etiopathogenesis of dental caries is complex, with several causes, and has not been completely clarified [1,2]. Currently, the principal etiological agent initiating dental caries is known to involve cariogenic bacteria, oral streptococci (mostly Streptococcus mutans), early stage carious lesions and lactobacilli from advanced lesions [1,3,4]. In parallel, the significance of factors initiating the carious process has been recognized (pathological factors), including, above all, free sugars in the diet, inadequate fluoride, poor oral hygiene and salivary dysfunction [5,6]. The physical and chemical properties of saliva are essential for the preservation of dental health [7]. However, knowledge of the proteins and glycoproteins present in saliva is only fragmentary. In our previous studies, we 
demonstrated a significant association between salivary acidic proline-rich proteins (APRP-1/2) and the severity of caries [8]. In turn, the mucin-glycoproteins present in saliva play a dominating role in the maintenance of oral health [9]. Currently, a heterogeneous group of 20 structurally-unique human mucins has been distinguished, from which the presence of MUC5B, MUC7, MUC19, MUC1 and MUC4 has been demonstrated in saliva $[9,10,11]$. MUC5B, MUC7 and MUC19 represent a subgroup of secreted mucins, while MUC1 and MUC4 represent membrane-associated mucins. Several data suggest the potential significance of salivary mucins in protection against dental caries [12,13,14,15]. Nevertheless, it remains to be established whether the manifestation of dental caries in adults is associated with quantitative disturbances in the above glycoproteins.

This study aimed at determining the relationship between dental caries in adults and levels of MUC5B and MUC7.

\section{Materials and methods}

\section{Patients selection}

The studies were conducted on 45 patients (25 40 years of age), where dental examination and calculation of the DMFT (decayed, missing and filled teeth) index, according to the World Health Organization (WHO) criteria [16], enabled the condition of dentition to be defined. This study was approved by the Bioethical Commission of Poznan University of Medical Sciences, Poland (No 60/15), in accordance with principles of good clinical practice (ISO 9001:2008) and the Helsinki Declaration [17].

Two research groups were distinguished: group 1 included 19 persons ( 25 - 37 years of age; mean 29.42 \pm 3.71 years; 9 men, 10 women) who were caries-free $(\mathrm{DMFT}=0)$. In no patients from this group were caries or fillings detected. Group 2 included 26 persons ( $25-40$ years of age; mean $32 \pm 5.74$ years; 13 men, 13 women) with severe dental caries (DMFT $>$ 13.9). Moreover, PL.I (Plaque Index) and GI (Gingival Index) were estimated. PL.I was calculated, representing an exponent of dental plaque presence and thickness [18]. In turn, GI was calculated on the basis of an evaluation of gingival health [19].

The patients qualified for the studies were healthy, with no general or chronic diseases in anamnesis. Moreover, the exclusion criteria included fungal infection in the oral cavity, destructive periodontal diseases, bruxism and smoking of cigarettes. In the three weeks preceding the study, the patients were not subjected to hygienization procedures or to the use of anti-bacterial mouth washes.

\section{Salivary sample collection}

Samples of saliva from the patients were collected between 8:00 AM and 11.00 AM. All subjects abstained from eating and drinking for $2 \mathrm{~h}$. Unstimulated whole saliva was collected for 10-15 min by the spitting method [20]. The volume of saliva samples obtained was $2 \mathrm{ml}$. Saliva samples were homogenized by vigorous shaking with the use of a vortex mixer and clarified by centrifugation at $3000 \times$ $\mathrm{g}$ for $15 \mathrm{~min}$ at $4^{\circ} \mathrm{C}$. The aliquots of clarified supernatants were stored at $-80^{\circ} \mathrm{C}$ for MUC5B and MUC7 measurements.

\section{Estimation of MUC5B and MUC7}

High sensitivity enzyme-linked immunosorbent sandwich assay (ELISA kits; Catalog No.: E0684h for MUC-5B and E1808h for MUC-7, EIAab; Wuhan, China) were used to determine the levels of MUC5B and MUC7 in the saliva samples. In the assay, recombinant MUC5B and recombinant MUC7 were used as standards (positive controls). The range of the standard curve was $0.312-20 \mathrm{ng} / \mathrm{ml}$ for MUC5B and $0.469-30 \mathrm{ng} / \mathrm{ml}$ for MUC7. The tests were performed as recommended by the manufacturer. Values of absorbance, depending on estimated MUC5B or MUC7, were read at the wavelength of $A=450 \mathrm{~nm}$ using a Reader 250 (bioMerieux). The results were obtained from standard curves. Every estimation of salivary MUC5B and MUC7 was repeated three times, and the obtained mean represented the individual result for the patient.

\section{Statistical analysis}

Results obtained in the studies were analyzed using Statistica v.13 software. In the analysis of quantitative characters, we used mean arithmetic values, standard deviations and median values. In the evaluation, we employed the nonparametric Mann-Whitney $U$ test. Moreover, the receiver operating characteristic (ROC) curve analysis was tested by DeLong method to define the relationship between the values of the studied mucins and demonstrated dental caries and to estimate the optimum cut-off value for detection of dental caries risk. Each point on the ROC curve represents a sensitivity/(1-specificity) pair corresponding to a particular decision threshold for the mucins. The area under the ROC curve (AUC) is a measure of how well a parameter (mucin) can distinguish between people with and without tooth decay. Additionally, to estimate the optimum cut-off value, in the graphs we presented the intersection point of the sensitivity and specificity values for the particular mucin. Differences 
with $p$-values higher than 0.05 , were considered insignificant.

\section{Results}

The studies were conducted on two groups of patients. No significant differences which could depend on age $(p=0.0891)$ or sex $(p=0.898)$ were revealed between the groups.

The obtained DMFT, PL.I and GI values in the two examined groups are shown in Table 1.

Table 1. DMFT, PL.I and GI values in caries-free patients (group 1 ) or those with severe caries (group 2)

\begin{tabular}{|c|c|c|c|}
\hline \multirow[t]{2}{*}{ Clinical indices } & $\begin{array}{c}\text { Group } 1 \\
(n=19)\end{array}$ & $\begin{array}{c}\text { Group } 2 \\
(n=26)\end{array}$ & \multirow[t]{2}{*}{$p$-values } \\
\hline & \multicolumn{2}{|c|}{ Mean values \pm SD [median values] } & \\
\hline DMFT & 0 & $\begin{array}{c}16.11 \pm 2.26 \\
{[15.50]}\end{array}$ & - \\
\hline PL.I & $\begin{array}{c}0.65 \pm 0.20 \\
{[0.70]}\end{array}$ & $\begin{array}{c}2.46 \pm 0.57 \\
{[2.15]}\end{array}$ & $p<0.0001$ \\
\hline GI & $\begin{array}{c}0.36 \pm 0.14 \\
{[0.40]}\end{array}$ & $\begin{array}{c}2.68 \pm 3.36 \\
{[2.05]}\end{array}$ & $p<0.0001$ \\
\hline
\end{tabular}

DMFT- decayed, missing and filled teeth index; PL.I - plaque index; GI- gingival index.

$p$-level of statistical significance in Mann-Whitney U test upon comparison of PL.I or GI values in group 2 vs. group 1 (control).

In none of the patients of group 1 were caries or fillings detected $(\mathrm{DMFT}=0)$, while the group 2 patients with severe dental caries manifested a mean DMFT value of $16.11 \pm 2.26$. In parallel, mean values of PL.I and GI amounted, respectively, in group 1 patients to $0.65 \pm 0.20 ; 0.36 \pm 0.14$ and in group 2 patients to $2.46 \pm$ $0.57 ; 2.68 \pm 3.36$. The data were significantly different in the studied groups.

Results of estimations representing concentrations of salivary mucins (MUC5B and MUC7) are shown in Table 2.

Table 2. Levels of salivary mucins MUC5B and MUC7 in caries-free patients (group 1) or those with severe caries (group 2)

\begin{tabular}{|c|c|c|c|}
\hline \multirow[t]{2}{*}{ Mucin } & $\begin{array}{c}\text { Group } 1 \\
(\mathrm{n}=19)\end{array}$ & $\begin{array}{c}\text { Group } 2 \\
(n=26)\end{array}$ & \multirow[t]{2}{*}{$p$-values } \\
\hline & \multicolumn{2}{|c|}{$\begin{array}{c}\text { Mean values } \pm \mathrm{SD} \text { [median values] } \\
\text { in } \mathrm{ng} / \mathrm{ml}\end{array}$} & \\
\hline MUC5B & $\begin{array}{c}0.63 \pm 0.35 \\
{[0.50]}\end{array}$ & $\begin{array}{c}0.38 \pm 0.32 \\
{[0.40]}\end{array}$ & $p=0.0233$ \\
\hline MUC7 & $\begin{array}{l}5.47 \pm 1.18 \\
\quad[5.60]\end{array}$ & $\begin{array}{l}1.39 \pm 0.86 \\
{[1.80]}\end{array}$ & $p<0.0001$ \\
\hline
\end{tabular}

In group 1, the mean concentrations of MUC5B and MUC7 amounted, respectively, to $0.63 \pm 0.35$ and $5.47 \pm 1.18 \mathrm{ng} / \mathrm{ml}$. In turn, in group 2 mean concentrations of MUC5B and MUC7 amounted to, respectively, $0.38 \pm 0.32$ and $1.39 \pm 0.86 \mathrm{ng} / \mathrm{ml}$. Upon comparison between the groups, group 2 demonstrated significantly lower levels of MUC5B and MUC7. At the same time, ROC was used for analysis of the obtained concentrations of MUC5B and MUC7 as related to the demonstrated caries curves.
The dental diagnosis of the presence of dental caries, or its absence, provided the criterion for sensitivity and specificity (Figures 1 and 2).

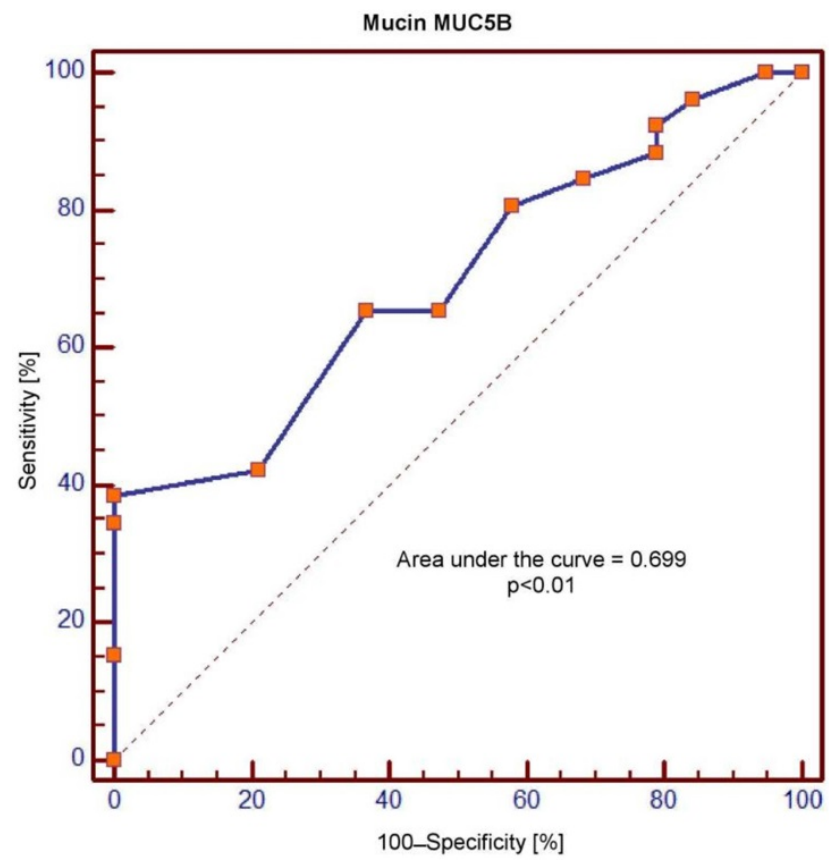

Figure 1. Receiver operating characteristic (ROC) curve for salivary levels of MUC5B

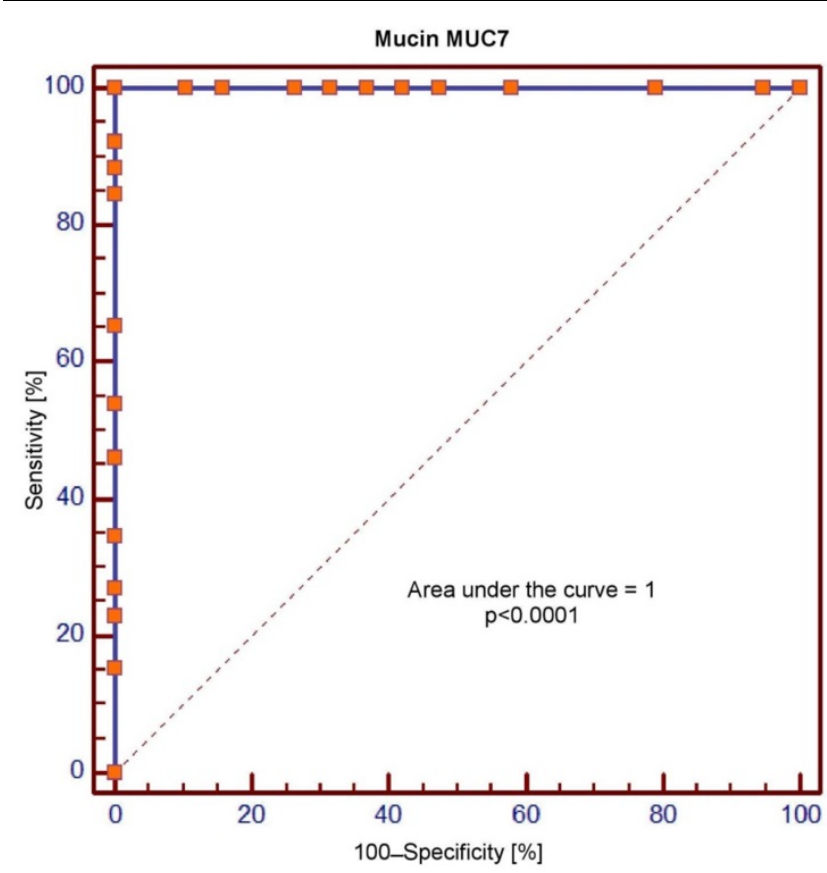

Figure 2. Receiver operating characteristic (ROC) curve for salivary levels of MUC7

The area under the curve (AUC) values for MUC5B and MUC7 amounted, respectively, to 0.699 and 1.0 and were significantly different $(p=0.0001)$. In the context of sensitivity/specificity, the obtained values within the range of 0 to 1 are presented in Figures 1 and 2. 
Crossing of the curves of sensitivity and specificity provided the cut-off point which, for MUC5B, amounted to $0.4 \mathrm{ng} / \mathrm{ml}$ (Figure 3). In turn, the cut-off point for MUC7 at a sensitivity of $=1$ and a specificity of $=1$ denotes the optimum (i.e. cut-off value corresponding to $100 \%$ sensitivity and specificity for the mucin, amounting to $2.5 \mathrm{ng} / \mathrm{ml}$ ) (Figure 4).
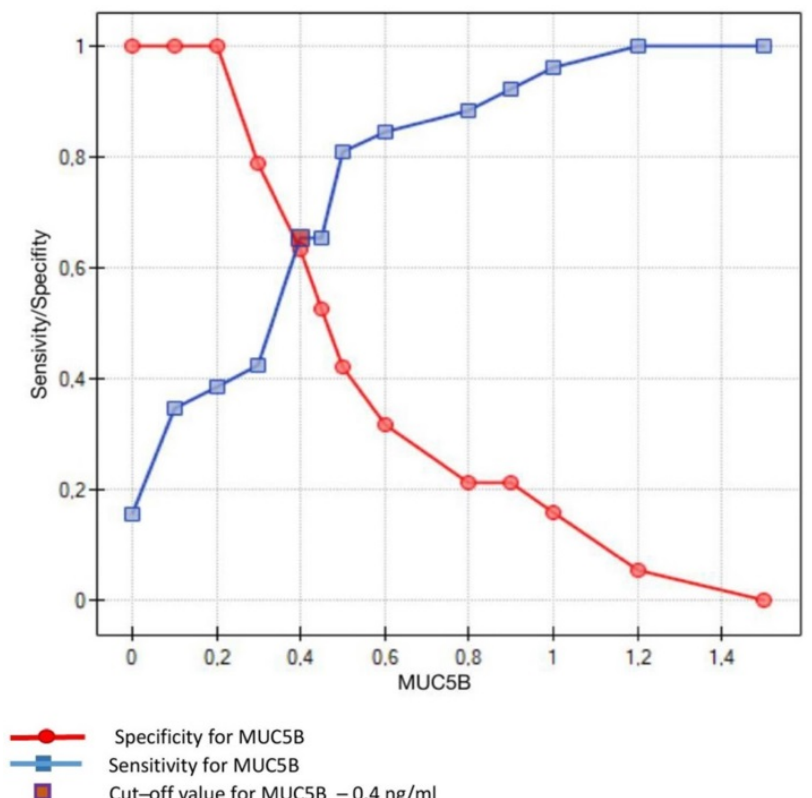

Specificity for MUC5B Sensitivity for MUC5B

Cut-off value for MUC5B $-0.4 \mathrm{ng} / \mathrm{ml}$

Figure 3. Sensitivity/Specificity of individual values of MUC5B in $\mathrm{ng} / \mathrm{ml}$ obtained in studied subjects (caries-free) and in patients with dental caries. Cut-off value for MUC5B is set at $0.4 \mathrm{ng} / \mathrm{ml}$

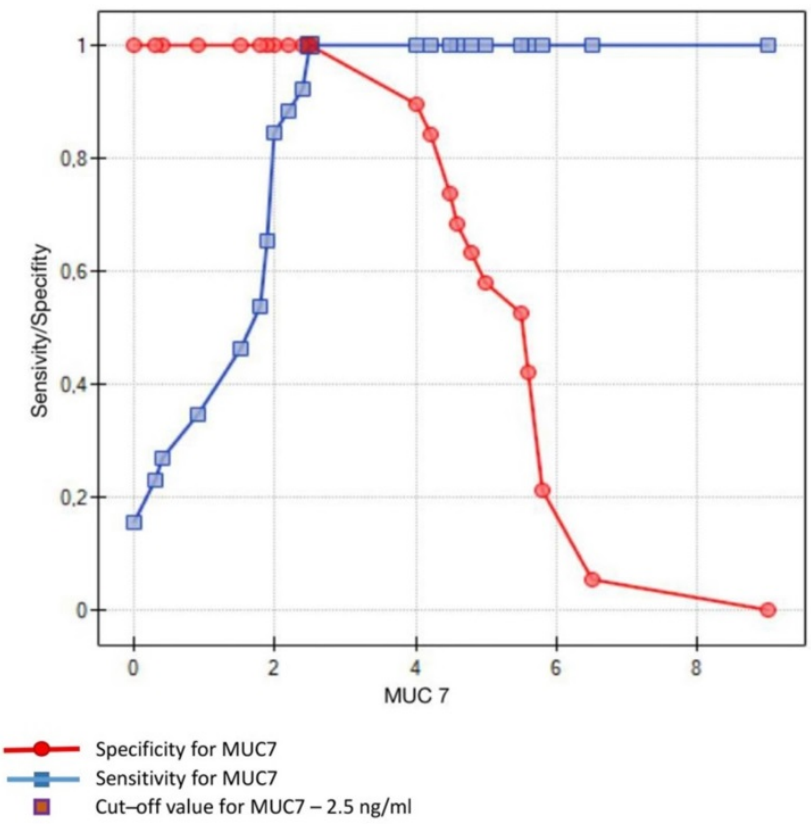

Figure 4. Sensitivity/Specificity of individual values of MUC7 in $\mathrm{ng} / \mathrm{ml}$ obtained in studied subjects (caries-free) and in patients with dental caries. Optimum value of cut-off for MUC7 is set at $2.5 \mathrm{ng} / \mathrm{ml}$

\section{Discussion}

Dental caries is a chronic disease, representing a pathological process involving a dissolution of hydroxyapatite and, through various stages of tissue destruction, potentially leading to a complete destruction of the tooth. In addition, a bacterial biofilm inducing these changes can cause even systemic diseases. Global population studies document that dental caries affects around 2.4 billion people worldwide, representing the most frequent civilization-linked disease $[1,21]$. In the multifactorial etiopathogenetic process of dental caries, an important role is played by salivary proteins including their antioxidants functions, and mucin-glycoproteins $[9,22]$. It has already been well documented that, in normal physiology, mucins are important components of innate immunity, protecting the mucosal surfaces from noxious physical, chemical, and biological influences. In parallel, it is indicated that salivary mucins protecting dental enamel may prevent dental caries [23]. Therefore, studies are justified which aim to clarify if development of dental caries in adults is linked to a disturbed content of mucins in resting saliva. In this study, we have compared concentrations of MUC5B and MUC7 in saliva of caries-free persons and in patients with severe dental caries. The study was conducted using high sensitivity and specificity ELISA tests for the detection of MUC5B or MUC7 in the saliva samples. Antibodies provided in the kits raised against a specific amino acid motif in MUC5B or MUC7. Earlier, it has been shown that carbohydrate-specific antibodies may not always identify the mucin due to local variations in glycosylation [24].

The MUC5B manifested in human saliva, with its large molecular weight, and the smaller - MUC7, are mainly secreted by submandibular/sublingual glands [9]. Both MUC5B and MUC7 have been identified within the mucus coat, protecting oral tissue surfaces [25]. At the same time, it has already been well documented that MUC5B manifesting high affinity to tooth hydroxyapatite contributes to the formation of the acquired enamel pellicle [26]. In in vitro studies, the mature (formed in the previous $72 \mathrm{~h}$ ) acquired enamel pellicle was found to provide protection to tooth enamel against demineralization and its prevailing component involves MUC5B $[27,28]$. Also, salivary MUC5B was found to significantly inhibit $S$. mutans attachment and biofilm formation on hydroxyapatite surfaces [23]. The unique effect of mucin action is thought to result from specific interaction between glycans on MUC5B with bacteria. In contrast to MUC5B, MUC7 manifests low affinity to tooth hydroxyapatite and it mainly remains in the solution phase of the oral tissue-saliva interface 
[15]. It is also indicated that MUC7 represents the primary mucin which exerts antimicrobial effect directly and preferentially against $S$. mutans $[23,29]$. Moreover, due to its bactericidal properties, the mucin exerts an effect on in vitro formation and reduction of the created S. mutans biofilm [14]. The mechanism of action of MUC7, which is distinct from that of MC5B, involves its direct interaction via sialic acid with oral microbes. Thus, both secreted mucins may prove important in the prevention of dental caries: MUC5B, by reducing $S$. mutans surface colonization; and MUC7 by direct inactivation of the cariogenic bacterium.

The results obtained in this study were analyzed in two distinct groups of adult patients. Group 1 included healthy, caries-free individuals $(\mathrm{DMFT}=0)$. In parallel, individuals in the group manifested good oral cavity hygiene and traits of benign gingivitis. In turn, group 2 included patients with severe caries (DMFT > 13.9), manifesting poor oral cavity hygiene and traits of moderate gingivitis. PL.I and GI values were significantly higher in group 2 than those in group 1, which additionally confirmed the difference in oral cavity health between the two groups of patients. In the conducted studies, we have demonstrated significantly higher levels of salivary MUC5B and MUC7 in group 1, which was caries-free than in group 2, in patients with severe dental caries. At the same time, analyzing the data obtained on the basis of the drawn ROC curves, a relationship was demonstrated between the demonstrated MUC5B values and MUC7 and the disclosed dental caries. However, only for MUC7 was the optimum cut-off (of $2.5 \mathrm{ng} / \mathrm{ml}$ ) obtained, above which the obtained results remained within the normal range. Thus, this parameter may prove clinically useful for diagnosis of the risk of dental caries development in generally healthy individuals. It is known that quantitative disorders of salivary mucins may be associated with the occurrence of chronic diseases. A significant increase in salivary levels of MUC5B and MUC7 in patients with Helicobacter pylori-associated gastric diseases [30] has already been well documented. Results obtained by us, presented for the first time in this study, enable the conclusion that reduced levels of salivary MUC5B and MUC7 can contribute to the development of caries in adults and, in addition, the demonstrated values of MUC7 may be useful in evaluation of the disease risk. Currently, the tests used for rapid risk assessment of dental caries are mainly based on the determinations of physicochemical properties of saliva such as hydration status, salivary viscosity, $\mathrm{pH}$, flow rate and buffering capacity. However, statistical studies show that none of the parameters mentioned above changes only in dental caries. On the other hand, the percentage of adults with caries in whom all the above parameters are changed is low and occurs in $35 \%$ of individuals [31]. It is also indicated that an increase in total antioxidant capacity (TAC) of saliva may be an indicator of active caries. However, the relevance of the relationship between the TAC of saliva and dental caries was found only in men [32]. In the context of these data, we have shown in our study a high specificity of MUC7 levels disturbances in saliva in patients with dental caries, so mucin may be a particularly valuable salivary factor for preventive dentistry. This conclusion is supported by the earlier studies of Banderas-Tarabayet et al. [33], who demonstrated, in patients with higher DMFT, a significant reduction or absence of high- and low-molecular-weight mucins as compared to subjects with lower DMFT. These results contrast with the data published by Gabryel-Porowska et al. [34] in which no significant differences were disclosed between salivary levels of MUC5B, and also MUC7 between patients with very low (control group) and moderate (research group) intensities of dental caries. However, the control group included just eight persons and it did not include caries-free patients, which resulted in a very wide and similar range of levels documented for the two mucins. Moreover, the estimated minimum values may induce doubts, since they were over two - fold lower than the minimum concentrations of MUC5B and MUC7 detected in the tests applied by the above cited authors. Therefore, it would be difficult to link those results to the results presented in this study. On the other hand, differentiated expression of salivary mucins in infant saliva, increased concentration of MUC5B and decreased level of MUC7 in the second half of the first year of life can be related to the phenomenon of eruption of primary dentition $[35,36]$.

In summary, we may conclude that the development of dental caries is linked to reduced concentrations of salivary mucins MUC5B and MUC7. In parallel, determinations of MUC7 levels seem to carry practical significance in the evaluation of the risk of development of the disease. However, this requires further studies on more numerous groups of patients.

\section{Acknowledgements}

The research was supported by the University of Medical Sciences in Poznan, Poland. Contract: 504-01-02206316-7/109-02658.

\section{Competing Interests}

The authors have declared that no competing interest exists. 


\section{References}

1. Pitts NB, Zero DT, Marsh PD, Ekstrand K, Weintraub JA, Ramos-Gomez F, et al. Dental caries. Nat Rev Dis Primers. 2017;3:17030. doi:10.1038/nrdp.2017.30.

2. Philip N, Suneja B, Walsh LJ. Ecological approaches to dental caries prevention: paradigm shift or shibboleth? Caries Res 2018; 52: 153-165.

3. Klein MI, Hwang G, Santos PH, Campanella OH, Koo H. Streptococcus mutans-derived extracellular matrix in cariogenic oral biofilms. Front Cell Infect Microbiol. 2015;5:10. doi:10.3389/fcimb.2015.00010.

4. Caufield PW, Schön CN, Saraithong P, Li Y, Argimón S. Oral lactobacilli and dental caries: a model for niche adaptation in humans. J Dent Res. 2015;94:110S-8S. doi:10.1177/0022034515576052.

5. Selwitz RH, Ismail AI, Pitts NB. Dental caries. Lancet. 2007;369: 51-9.

6. Forssten SD, Björklund M, Ouwehand AC. Streptococcus mutans, caries and simulation models. Nutrients. 2010;2:290-98.

7. Dawes C, Pedersen AM, Villa A, Ekström J, Proctor GB, Vissink A, et al. The functions of human saliva: a review sponsored by the World Workshop on Oral Medicine VI. Arch Oral Biol. 2015;60:863-74. doi:10.1016/j.archoralbio.2015.03.004.

8. Szkaradkiewicz-Karpińska AK, Zeidler A, Goślińska-Kuźniarek O, Uram K, Szkaradkiewicz A. Oral lactobacilli and salivary acidic proline-rich proteins (APRP-1/2) in dental caries. J Physiol Pharmacol. 2018;69:139-44.

9. Tabak LA. In defense of the oral cavity: structure, biosynthesis, and function of salivary mucins. Annu Rev Physiol. 1995;57:547-64.

10. Liu B, Lague JR, Nunes DP, Toselli P, Oppenheim FG, Soares RV, et al. Expression of membrane-associated mucins MUC1 and MUC4 in major human salivary glands. J Histochem Cytochem. 2002;50:811-20.

11. Culp DJ, Robinson B, Cash MN, Bhattacharyya I, Stewart C, Cuadra-Saenz G. Salivary mucin 19 glycoproteins: innate immune functions in Streptococcus mutans-induced caries in mice and evidence for expression in human saliva. J Biol Chem. 2015;290:2993-3008. doi:10.1074/jbc.M114.597906.

12. Baughan LW, Robertello FJ, Sarrett DC, Denny PA, Denny PC. Salivary mucin as related to oral Streptococcus mutans in elderly people. Oral Microbiol Immunol. 2000;15:10-14.

13. Leone CW, Oppenheim FG. Physical and chemical aspects of saliva as indicators of risk for dental caries in humans. J Dent Educ. 2001;65:1054-62.

14. Wei GX, Campagna AN, Bobek LA. Effect of MUC7 peptides on the growth of bacteria and on Streptococcus mutans biofilm. J Antimicrob Chemother. 2006;57:1100-109.

15. Frenkel ES, Ribbeck K. Salivary mucins in host defense and disease prevention. J Oral Microbiol. 2015;7:29759. doi:10.3402/jom.v7.29759.

16. World Health Organization. Oral Health Surveys: Basic Methods. Geneva. 2013.p.42-47.

17. World Medical Association Declaration of Helsinki: ethical principles for medical research involving human subjects. World Medical Association. JAMA. 2013;310:2191-219.

18. Silness J, Löe H. Periodontal disease in pregnancy. II. Correlation between oral hygiene and periodontal condition. Acta Odontol Scand. 1964;22:121-35.

19. Löe $\mathrm{H}$. The gingival index, the plaque index and the retention index system. J Periodontol. 1967;38:610-16.

20. Bhattarai KR, Kim H-R, Chae H-J. Compliance with saliva collection protocol in healthy volunteers: strategies for managing risk and errors. Int J Med Sci. 2018;15:823-831.

21. Bagramian RA, Garcia-Godoy F, Volpe AR. The global increase in dental caries. A pending public health crisis. Am J Dent. 2009;22:3-8.

22. Szkaradkiewicz-Karpińska AK, Sak M, Goślińska-Kuźniarek O, Sokalski J, Szkaradkiewicz A. Human salivary acidic proline-rich proteins (APRP-1/2) in adult patients with dental caries. Dentistry. 2017;7:437. doi:10.4172/2161-1122.1000437

23. Frenkel ES, Ribbeck K. Salivary mucins protect surfaces from colonization by cariogenic bacteria. Appl Environ Microbiol. 2015;81:332-38.

24. Sonesson M, Wickström C, Kinnby B, Ericson D, Matsson L. Mucins MUC5B and MUC7 in minor salivary gland secretion of children and adults. Arch Oral Biol. 2008;53:523-527.

25. Linden SK, Sutton P, Karlsson NG, Korolik V, McGuckin MA. Mucins in the mucosal barrier to infection. Mucosal Immunol. 2008;1:183-97.

26. Lindh L, Glantz PO, Carlstedt I, Wickström C, Arnebrant T. Adsorption of MUC5B and the role of mucins in early salivary film formation. Colloids Surf B Biointerfaces. 2002;25:139-46.

27. Gibbins HL, Proctor GB, Yakubov GE, Wilson S, Carpenter GH. Concentration of salivary protective proteins within the bound oral mucosal pellicle. Oral Dis. 2014;20:707-13.

28. Gibbins HL, Yakubov GE, Proctor GB, Wilson S, Carpenter GH. What interactions drive the salivary mucosal pellicle formation? Colloids Surf B Biointerfaces. 2014;120:184-92.

29. Ge J, Catt DM, Gregory RL. Streptococcus mutans surface alpha-enolase binds salivary mucin MG2 and human plasminogen. Infect Immun. 2004:72:6748-52.

30. Silva DG, Stevens RH, Macedo JMB, Hirata R, Pinto AC, Alves LM, et al. Higher levels of salivary MUC5B and MUC7 in individuals with gastric diseases who harbor Helicobacter pylori. Arch Oral Biol. 2009;54:86-90.

31. Gopinath VK, Arzreanne AR. Saliva as a diagnostic tool for assessment of dental caries. Arch Orofac Sci. 2006;1:57-59.
32. Ahmadi-Motamayel F, Goodarzi MT, Hendi SS, Kasraei S, Moghimbelgi A. Total antioxidant capacity of saliva and dental caries. Med Oral Patol Oral Cir Bucal. 2013;18:553-6.

33. Banderas-Tarabay JA, Zacarías-D'Oleire IG, Garduño-Estrada R, Aceves-Luna E, González-Begné M. Electrophoretic analysis of whole saliva and prevalence of dental caries. A study in Mexican dental students. Arch Med Res. 2002;33:499-505.

34. Gabryel-Porowska H, Gornowicz A, Bielawska A, Wójcicka A, Maciorkowska E, Grabowska Sz, et al. Mucin levels in saliva of adolescents with dental caries. Med Sci Monit. 2014;20:72-77.

35. Ruhl S, Rayment SA, Schmalz G,Hiller KA, Troxler RF. Proteins in whole saliva during the first year of infancy. J Dent Res. 2005;84:29-34.

36. Manconi B, Cabras T, Pisano E, Sanna MT, Olianas A, Fanos V et al. Modifications of the acidic soluble salivary proteome in human children from birth to the age of 48 months investigated by a top-down HPLC-ESI-MS platform. J Proteomics. 2013;91:536-43. 